\title{
Paramagnetic and fluorescent liposomes for target-specific imaging and therapy of tumor angiogenesis
}

\author{
Gustav J. Strijkers • Ewelina Kluza • Geralda A. F. Van Tilborg • \\ Daisy W. J. van der Schaft • Arjan W. Griffioen • \\ Willem J. M. Mulder $\cdot$ Klaas Nicolay
}

Received: 1 March 2010/Accepted: 24 March 2010/Published online: 14 April 2010

(C) The Author(s) 2010. This article is published with open access at Springerlink.com

\begin{abstract}
Angiogenesis is essential for tumor growth and metastatic potential and for that reason considered an important target for tumor treatment. Noninvasive imaging technologies, capable of visualizing tumor angiogenesis and evaluating the efficacy of angiostatic therapies, are therefore becoming increasingly important. Among the various imaging modalities, magnetic resonance imaging (MRI) is characterized by a superb spatial resolution and anatomical soft-tissue contrast. Revolutionary advances in contrast agent chemistry have delivered versatile angiogenesis-specific molecular MRI contrast agents. In this paper, we review recent advances in the preclinical application of paramagnetic and fluorescent liposomes for noninvasive visualization of the molecular processes involved in tumor angiogenesis. This liposomal contrast
\end{abstract}

G. J. Strijkers $(\bowtie) \cdot$ E. Kluza $\cdot$ K. Nicolay

Biomedical NMR, Department of Biomedical Engineering,

Eindhoven University of Technology, PO Box 513, 5600 MB

Eindhoven, The Netherlands

e-mail: g.j.strijkers@tue.nl

\section{G. A. F. Van Tilborg}

Image Sciences Institute, University Medical Center Utrecht, Utrecht, The Netherlands

D. W. J. van der Schaft

Soft Tissue Biomechanics and Engineering, Department of Biomedical Engineering, Eindhoven University of Technology, Eindhoven, The Netherlands

\footnotetext{
A. W. Griffioen

Angiogenesis Laboratory, Department of Medical Oncology,

VU University Medical Center, Amsterdam, The Netherlands

W. J. M. Mulder

Translational and Molecular Imaging Institute, Department of Radiology, Mount Sinai School of Medicine, New York, NY, USA
}

agent platform can be prepared with a high payload of contrast generating material, thereby facilitating its detection, and is equipped with one or more types of targeting ligands for binding to specific molecules expressed at the angiogenic site. Multimodal liposomes endowed with contrast material for complementary imaging technologies, e.g., MRI and optical, can be exploited to gain important preclinical insights into the mechanisms of binding and accumulation at angiogenic vascular endothelium and to corroborate the in vivo findings. Interestingly, liposomes can be designed to contain angiostatic therapeutics, allowing for image-supervised drug delivery and subsequent monitoring of therapeutic efficacy.

Keywords Angiogenesis - Angiostatic therapy · Magnetic resonance imaging - Molecular imaging . Liposomes - Synergistic targeting - Tumor

\section{Introduction}

Inhibition of tumor angiogenesis is a modern and popular approach to fight tumor progression [1, 2]. Therapeutic interventions aimed at reducing tumor growth by angiogenesis inhibition are rapidly finding their way into clinical practice [2]. The origin of this approach is that angiogenesis inhibitors target cells that support tumor growth, i.e., endothelial cells, instead of targeting the tumor cells directly. Endothelial cells are genetically more stable than tumor cells and thus less likely to develop drug resistance. Moreover, the endothelial cells are directly accessible from the bloodstream, enabling nanocarriers with a high payload of angiostatic drugs to reach their target site efficiently. There are several classes of angiostatic drugs, e.g., growth factor blockers [3], growth factor signaling inhibitors, 
extracellular matrix modulators [4], and endothelial cell proliferation and migration inhibitors [5-7]. In 2004, the Food and Drug Administration approved the first angiostatic drug (Avastin) for human application [8].

These developments call for new noninvasive readouts to test the efficacy of novel angiostatic drugs. In a preclinical setting, different methods are available to monitor changes in angiogenic activity during the course of treatment. Typically, new agents are first evaluated in vitro before further assessment in animal models. Inhibition or regression of the volume of a subcutaneously implanted tumor can be estimated by an external morphological measurement, e.g., using a caliper. Such measurements are often inaccurate, as they do not account for inward growth, irregular tumor shape with multiple lobes and development of metastases. Moreover, morphological changes often occur late after initiation of the angiostatic treatment and are therefore a poor readout of early drug efficacy. The effects of the new drug on the tumor vasculature can be assessed by ex vivo analysis of the tumor microvessel density (MVD). Several angiogenesis inhibitors, including endostatin and anginex (Anx), have shown to decrease the tumor MVD, which is therefore considered a parameter that may be used to predict therapeutic effect $[5,9]$. A major drawback of the MVD readout is that-as with all histological analyses-it can only be determined with invasive and laborious procedures following staining of ex vivo tumor tissue sections, making it difficult to assess tumor vessel density heterogeneity and precluding longitudinal assessment during the course of treatment. Therefore, noninvasive imaging methods that report on tumor angiogenesis are highly desired, since methods to visualize and quantify angiogenic activity in vivo would allow longitudinal monitoring and thus facilitate the early evaluation of a given angiostatic therapy. For this purpose, magnetic resonance imaging (MRI) is a prime candidate.

MRI is a powerful noninvasive imaging modality capable of generating high-resolution images with superb soft tissue contrast. Already, MRI offers a broad spectrum of techniques to localize, visualize and characterize tumors. For example, anatomical $\mathrm{T}_{2}$-weighted images help to define anatomical borders of solid tumors with outstanding resolution and contrast, and aid in distinguishing and classifying healthy from diseased tissue [10]. Also MR spectroscopy can be used to assess tumor $\mathrm{pH}$ and metabolic activity [11]. MR image contrast reflecting endogenous cellular protein and peptide content in the tumor can be produced by selective radiofrequency labeling of the protein and peptide amide protons and exploiting the magnetization transfer to water via hydrogen exchange [12]. Recent advances in whole-body diffusion-weighted imaging show promise for diagnosing lesions throughout the entire human body as well as for assessing lymph node metastases, with superior spatial resolution, and sensitivity and specificity that rival FDG-PET imaging [13]. In addition, hemodynamic parameters such as the fractional blood volume, vascular permeability, tumor blood perfusion, and lymphatic drainage can be assessed using contrastenhanced MRI [14-16].

Recent advances in molecular imaging - the field that aims to noninvasively visualize processes at the molecular and cellular level-have broadened the use of MRI in oncology [16-18]. Sophisticated contrast agents have been developed, equipped with targeting ligands directed toward angiogenesis-specific markers, which allow for noninvasive in vivo visualization of the angiogenic activity in the tumor. A variety of targeted Gd-, ironoxide-, and fluorinebased MRI contrast agents have been developed for this purpose, ranging from low molecular weight agents to nanoparticulate agents of different size and composition [19-30].

In this paper, we review our recent work on the use of multimodal liposomes for target-specific preclinical imaging of tumor angiogenesis and therapy. Over the last decades, liposomes were extensively studied as drug carriers for cancer therapy [31]. Encapsulation of the drug in a liposome improves the pharmacokinetic profile, thereby increasing the amount of drug effectively delivered to the tumor, while preventing rapid clearance, drug degradation, and inactivation. When equipped with a high payload of contrast generating material and conjugated with one or more types of targeting ligands, liposomes turn into a potent contrast agent for tumor angiogenesis imaging. Often, the liposomes are endowed with a contrast agent for a complementary imaging modality, e.g., fluorescence imaging, for validation purposes and to investigate binding and accumulation at the cellular level. Combined with angiostatic drugs, these multimodal liposomes allow for image-supervised drug delivery and monitoring of angiostatic therapy.

\section{Multimodal liposomes for visualization of tumor angiogenesis}

A liposome is a spherical vesicle composed of a bilayer of naturally occurring phospholipids or closely related synthetic amphiphiles enclosing an aqueous interior [32, 33]. For in vivo application purposes, the liposome should be biocompatible, stable, and possess favorable pharmacokinetic properties. Therefore, liposomes are usually comprised of components that energetically favor a bilayer organization, such as phospholipids comprising a polar head group and two fatty acyl chains [33]. For additional stabilization, cholesterol is commonly included [33]. To reduce interactions with blood circulating proteins and 
cells polyethyleneglycol-lipids (PEG-lipids) may be incorporated, which increases the liposome blood circulation half-life and ensures optimal pharmacokinetics [34, 35].

Liposomes are commonly prepared by a lipid film hydration technique. The preparation involves several steps. First, the appropriate amounts of lipids are dissolved and mixed in an organic solvent, e.g., chloroform or chloroform/methanol. The organic solvent is then removed by rotary evaporation yielding a thin lipid film on the sides of a round bottom flask. Next, the lipid film is hydrated by addition of an aqueous medium at a temperature above the lipid gel-liquid crystal transition temperature, resulting in a solution of multilamellar lipid vesicles. Finally, the multilamellar vesicles are downsized by extrusion of the lipid suspension through a polycarbonate filter of well-defined pore size at elevated temperature or by sonication to yield a suspension of liposomes with a narrow and well-defined size distribution.

Initially, MRI contrast properties were introduced in liposomes by enclosing low molecular weight Gd- and Mnbased contrast agent in the aqueous interior [36-44]. The applicability of these liposomal MRI contrast agents was, however, limited because entrapment of the $\mathrm{Gd}$ and $\mathrm{Mn}$ effectively leads to very low relaxivity - the parameter that defines the potency of the agent to introduce contrast in the MR images-due to limited water exchange across the liposomal bilayer. Moreover, the entrapped contrast agent may leak out. An alternative approach is therefore to include $\mathrm{Gd}$ entities in the liposomal bilayer. A number of Gd-containing amphiphiles have been developed for this purpose [45-49]. We introduced a paramagnetic liposome of which the schematic design is shown in Fig. 1 [50]. The liposomal bilayer consisted of DSPC, PEG $_{2000}$-DSPE, maleimide-PEG 2000 -DSPE, cholesterol, and $25 \mathrm{~mol} \% \mathrm{Gd}-$ DTPA-di(stearylamide). The presence of this high payload of Gd-containing lipids ensures a high particulate relaxivity. Half of the Gd-DTPA-di(stearylamide) is contained in the outer bilayer with free access to solute water and therefore the relaxivity is much less exchange limited. Fluorescent properties were introduced by addition of $0.1 \mathrm{~mol} \%$ rhodamine PE. The size of these liposomes was $120 \mathrm{~nm}$. In a follow-up study, these liposomes were further characterized concerning morphology and relaxivity [51]. Hak et al. [52] have recently designed a liposome containing Gd-DOTA-DSPE which, in contrast to Gd-DTPAdi(stearylamide), exhibited no detectable transmetallation upon exposure to $\mathrm{Zn}$ ions in the presence of phosphate ions. This is an important improvement to the safety of the agent, as transmetallation in the presence of phosphate anions has been proposed as a likely mechanism underlying nephrogenic systemic fibrosis (NSF), a severe disorder associated with the use of Gd-based contrast agents in patients with end-stage renal disease [53]. Additionally, liposomes with Gd-DOTA-DSPE displayed a considerable higher relaxivity [52].

Specificity for tumor angiogenesis can be introduced by decorating the exterior of the liposomes with suitable targeting ligands, e.g., antibodies or peptides, as schematically illustrated in Fig. 1. Covalent binding of the targeting ligands to the liposome is achieved by coupling to lipids with a functional reactive group, such as maleimide, thiol, amine, or carboxylic acid. Targeted liposomal MRI contrast agent can be used to follow the effects of therapeutic interventions, as will be discussed in detail further on. Imaging and therapy may be combined by inclusion of drugs in the liposomal formulation. Water-soluble drug can be enclosed in the liposomal interior. Drugs may also be enclosed in the liposomal bilayer or attached to the distal end of the PEG chains (Fig. 1).

\section{Multimodal imaging of tumor angiogenesis and angiostatic therapy}

Many studies, which aimed at in vivo visualization of the molecular hallmarks of tumor angiogenesis, have focused on the $\alpha_{v} \beta_{3}$ integrin $[19,20,29,30,54-63]$. The $\alpha_{v} \beta_{3}$ integrin is strongly expressed on the activated endothelium of angiogenic blood vessels and is involved in many tumor related processes. Importantly, the integrin is only weakly expressed on resting endothelial cells in nondiseased tissue, making it an attractive specific target for tumor angiogenesis imaging [64]. MR imaging of tumor angiogenesis using a targeted paramagnetic vesicles was pioneered by Sipkins et al. [19]. They demonstrated enhanced and detailed imaging of the $\alpha_{\mathrm{v}} \beta_{3}$ expressing angiogenic vasculature in rabbit carcinomas after intravenous administration of the aforementioned agent, providing a noninvasive means to assess the growth and malignant phenotype of tumors. The $\alpha_{\mathrm{v}} \beta_{3}$ integrin binds to a wide variety of extracellular matrix proteins, which expose the tripeptide sequence Arg-Gly-Asp (RGD) as a common receptor recognition signal [65]. This peptide is used as a targeting moiety for angiogenesis targeting and several isoforms that include the tripeptide sequence have been developed. A cyclic conformation (cyclic RGD) has favorable binding properties when compared to a linear peptide and multimers of the RGD peptide have been shown to increase the affinity and specificity [56, 57, 66].

A bimodal, MRI and fluorescence, approach to tumor angiogenesis imaging was applied by Mulder et al. [22]. As a bimodal contrast agent, the above-described liposomes were used, which have a high payload of Gd-containing lipid for MRI purposes and rhodamine fluorescent lipids for optical imaging. The 150-nm-diameter liposomes were 


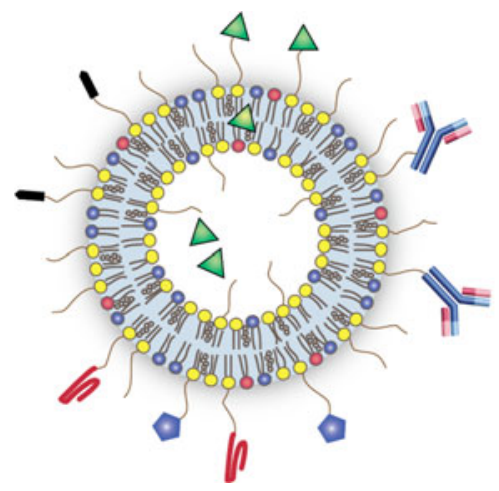

Fig. 1 Schematic representation of a target-specific multimodal liposome for combined angiogenesis imaging and therapy. Generally, the liposome contains a high payload of Gd containing lipids and fluorescent lipids for MRI and fluorescence microscopy, respectively. The liposome can be equipped with single or multiple populations of

conjugated with on average about 700 cyclic RGD peptides per particle to introduce specificity for the $\alpha_{\mathrm{v}} \beta_{3}$ integrin. The high number of RGD moieties attached to the liposomes ensures a high affinity for the target on the basis of multivalency.

The targeting and multimodal-imaging concept was first tested in vitro using human endothelial cells (human umbilical vein endothelial cells). Cells incubated with specific RGD-conjugated liposomes displayed bright rhodamine fluorescence, which was lacking in case of control incubations with nonspecific RAD-conjugated liposomes. Detailed analysis showed that the RGD-conjugated liposomes were internalized in the perinuclear region of the cells. MR imaging of cell pellets revealed a large contrast between specific and control incubations. Application of RGD-conjugated liposomes resulted in a substantial $T_{1}$ relaxation time shortening of the cell pellets. These results proved specificity and high affinity for endothelial cells. The mechanism of RGD-conjugated liposome uptake and the effect of internalization on the relaxation properties were further investigated in two recent studies [67, 68]. A dramatic lowering of the longitudinal relaxation rate $r_{1}$ (relaxivity quenching) for cell-internalized targeted liposomes was observed that warrants caution in the quantitative interpretation of the observed MRI signal.

In vivo angiogenesis imaging was performed on subcutaneous xenograft human LS174T colon carcinoma tumors in athymic mice (Fig. 2). After initial localization of the tumor on the right flank of the mouse using a $\mathrm{T}_{2^{-}}$ weighted scan, repeated high-resolution $\mathrm{T}_{1}$-weighted images were recorded pre- and post-intravenous injection of RGD-conjugated liposomes (Fig. 2a). A pixel-by-pixel comparison of pre- and post-injection images, revealed those pixels that were significantly enhanced by the paramagnetic liposomes. After injection of RGD-conjugated liposomes, the pixels that showed enhancement were

fluorescent lipid $\quad$ PEG-phospholipid

antibodies or peptides to introduce target specificity, biotin for an avidin-induced clearance strategy, as well as with drugs in the lumen, in the bilayer or by covalent binding to the distal ends of the PEG chains

mainly located in the rim of the tumor (Fig. 2a), whereas for injections with nonspecific RAD-conjugated liposome enhancement was more evenly distributed through the tumor volume (Fig. 2b). Injection of RGD-conjugated liposomes did not lead to a significant amount of enhanced pixels when the mice were pretreated with MRI-invisible nonparamagnetic RGD-conjugated liposomes to block the $\alpha_{v} \beta_{3}$ binding of paramagnetic RGD-conjugated liposomes (Fig. 2c).

The in vivo MRI findings as shown in Fig. 2 strongly suggested that RGD-conjugated liposomes predominantly accumulated in the tumor through a specific interaction. Proof for exclusive association with the angiogenic endothelium of the tumor blood vessels, however, was not provided by the in vivo experiments. Here, the true power of a multimodal contrast agent comes into play, in this case combining the in vivo MRI findings with ex vivo fluorescence microscopy. Fluorescence microscopy revealed distinct differences in the localization of the liposomes in the tumors of mice injected with RGD- or RAD-conjugated liposomes. RGD-conjugated liposomes were found exclusively associated with tumor blood vessels (Fig. 3a, b), suggesting specific association with $\alpha_{\mathrm{v}} \beta_{3}$ integrin expressed on the endothelial cells. In the center of the tumor (Fig. 3c) almost no liposomal fluorescence was observed, in agreement with the in vivo MRI findings. Fluorescence from the RAD-conjugated liposomes was observed more diffusely spread beyond the vasculature of the tumor (Fig. 3d), indicative for nonspecific extravasation. Extravasation of RAD-conjugated liposomes was further facilitated by a longer circulation time when compared to RGDconjugated liposomes [69].

In a follow-up study, the above $\alpha_{v} \beta_{3}$ integrin specific RGD-conjugated liposomes were used for noninvasive evaluation of the efficacy of angiogenesis inhibitors during the course of therapy [24]. Again, in vivo MRI and ex vivo 


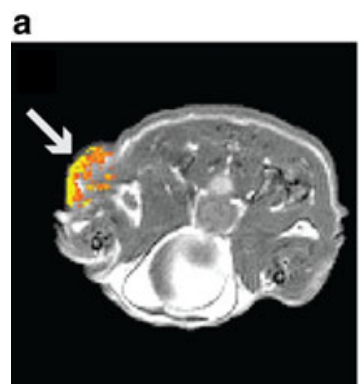

b

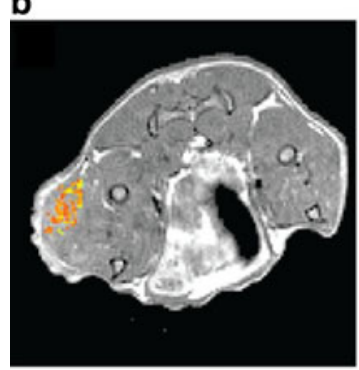

c

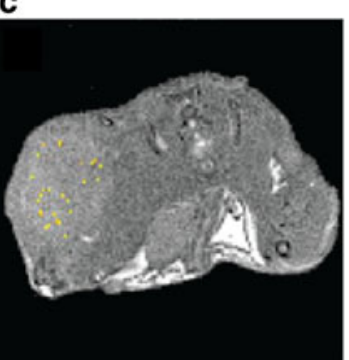

RGD

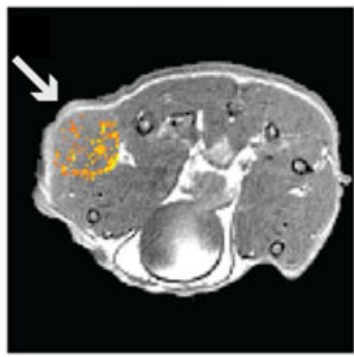

RAD

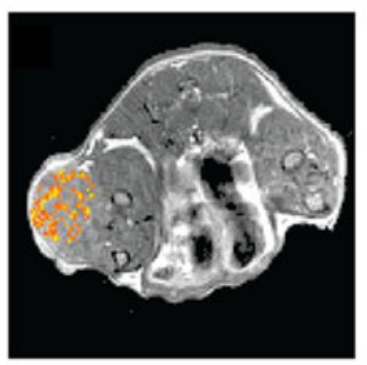

RGD competition

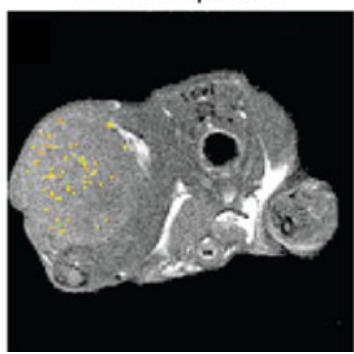

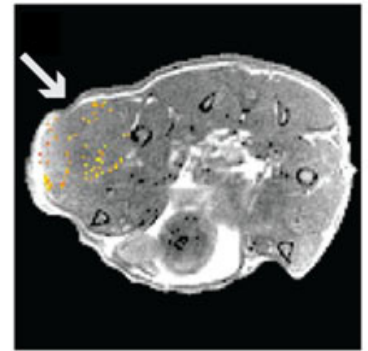

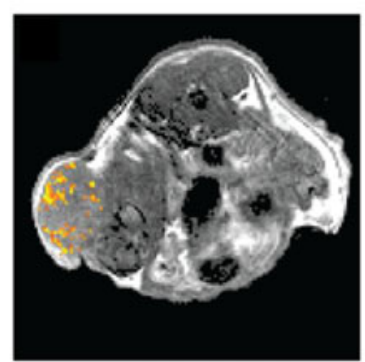

$40 \%$
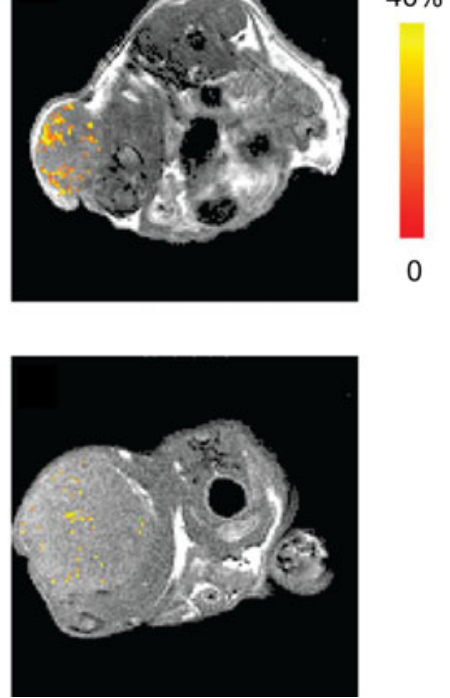

Fig. 2 In vivo visualization of tumor angiogenesis by application of $\alpha_{\mathrm{v}} \beta_{3}$ integrin-targeted paramagnetic and fluorescent liposomes. a MR images of three slices through a mouse with a xenograft human LS174T colon carcinoma in a subcutaneous location on the right flank. The images were taken $35 \mathrm{~min}$ after injection of RGDconjugated liposomes, which target the $\alpha_{\mathrm{v}} \beta_{3}$ integrins expressed on the angiogenic tumor endothelium. Pixels in the tumor that were significantly enhanced by the presence of the paramagnetic liposomes were highlighted and color coded according to the scale on the right. Enhancement was mainly found at the rim of the tumor in correspondence with the spatial distribution of angiogenic blood

fluorescence microscopy using biomodal RGD-conjugated liposomes were fully exploited to image ongoing angiogenesis during the treatment. B16F10 mouse melanoma tumors were grown on the flank of C57BL/6 mice. Angiostatic therapy consisted of treatment for 3 or 14 days with either endostatin or anginex (Anx). The percentage of enhanced pixels in the tumor after injection with paramagnetic and fluorescent RGD-conjugated liposomes served as a noninvasive in vivo readout of angiogenic activity (Fig. 4b). Ex vivo fluorescence microscopy proved specific association with the tumor blood vessels. As a gold standard for assessing the angiogenic activity, the microvessel density (MVD) was determined by ex vivo staining tumor blood vessels with CD31 antibody and counting their density (Fig. 4a). MVD revealed a significant treatment vessels. The percentage of enhanced pixels serves as a noninvasive readout of angiogenic activity. b MR images of a mouse 35 min after injection with nonspecific RAD-conjugated liposomes. Nonspecific liposomes distributed more evenly throughout the whole tumor. c MR images of a mouse $35 \mathrm{~min}$ after pretreatment with nonparamagnetic RGD-conjugated liposomes to block the $\alpha_{v} \beta_{3}$ integrin followed by injection with paramagnetic RGD-conjugated liposomes. Only a small number of pixels showed signal enhancement proving specificity of the $\alpha_{v} \beta_{3}$ targeting concept. Adapted from Ref. [22] with permission

effect for anginex (3 and 14 days treatment) and endostatin (3 days treatment). Importantly, the in vivo MRI readout of angiogenic activity closely reflected the treatment effects as deduced from the ex vivo analyses, proving that the multimodal liposomes can be used to noninvasively monitor efficacy of angiostatic therapy.

\section{Improving target specificity by avidin-induced clearance of circulating liposomes}

The RGD-conjugated liposomes for imaging angiogenesis, as described in the previous section, were designed to exhibit a long blood circulation half-life, which was achieved by including PEG-lipids in the liposomal bilayer. 
Fig. 3 Fluorescence microscopy of dissected tumors of mice, which were injected with paramagnetic, fluorescent, RGD- or RAD-conjugated liposomes. a, b In the rim of the tumors, the red rhodamine fluorescence originating from the RGD-conjugated liposomes revealed circular and longitudinal distribution patterns associated with blood vessels. Cell nuclei were stained with DAPI (blue fluorescence). c A slice through the middle of the tumor revealed no fluorescence from RGDconjugated liposomes, in agreement with a lack of angiogenic blood vessels in this location. d A diffuse pattern of fluorescence was observed in tumors of mice injected with nonspecific RAD-conjugated liposomes, indicative for nonspecific distribution beyond the blood vessels throughout the whole tumor. Adapted from Ref. [22] with permission
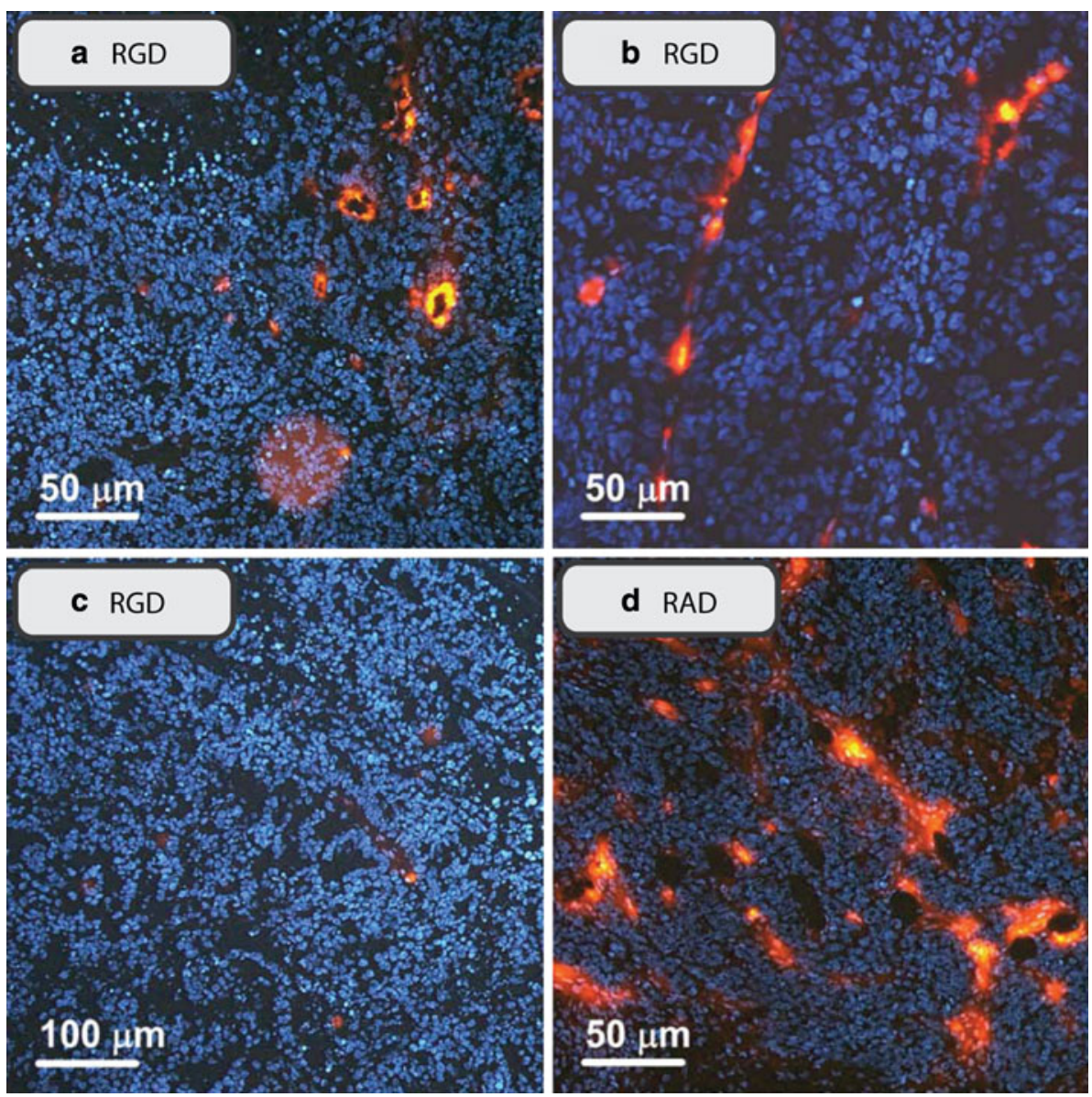

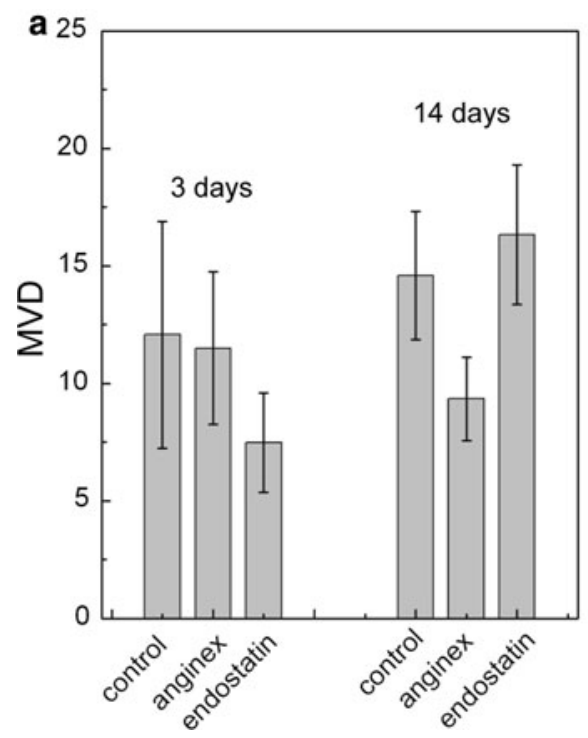

Fig. 4 In vivo assessment of angiostatic therapy efficacy by targeted multimodal liposomes. Mice with a B16F10 melanoma tumor in a subcutaneous position on the right flank were treated for 3 or 14 days with either anginex or endostatin, which both are angiostatic compounds. a Microvessel density (MVD, mean number of vessels per $0.25 \mathrm{~mm}^{2}$ ) was determined by ex vivo staining of tumor blood vessels with CD31 antibody and counting their density. (b) The percentage of enhanced pixels in the tumor on $\mathrm{T}_{1}$-weighted MRI after

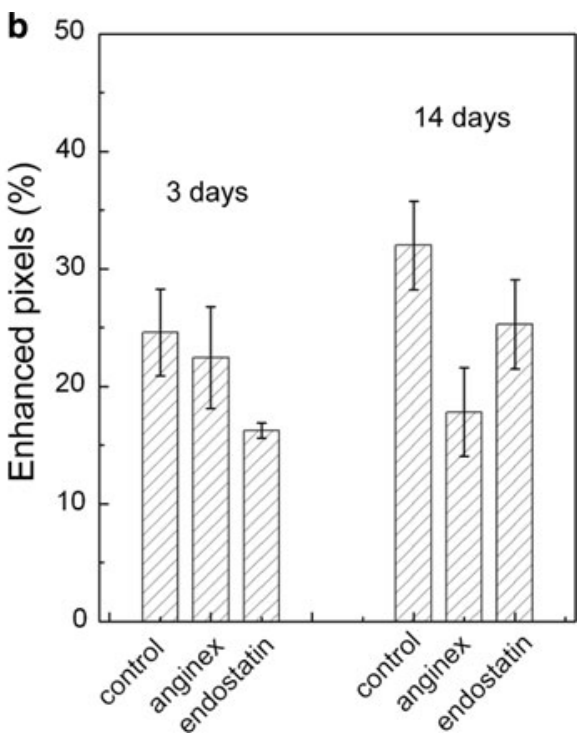

injection with paramagnetic RGD-conjugated liposomes served as a noninvasive in vivo readout of angiogenic activity (see also Fig. 2). MVD revealed a significant treatment effect for anginex (3 and 14 days treatment) and endostatin ( 3 days treatment). Importantly, the in vivo MRI readout of angiogenic activity closely reflected the treatment effects as deduced from the ex vivo analyses, proving that the multimodal liposomes can be used to noninvasively monitor angiostatic therapy. Adapted from Ref. [24] with permission 
On the one hand, a long circulation half-life is potentially advantageous for molecular imaging as it may increase the amount of contrast agent that accumulates at the angiogenic site enabled by prolonged time for interaction with the targets. This is particularly necessary for MRI, since this is a relatively insensitive technique for the detection of low concentrations of contrast agent. On the other hand, the MR images will suffer from undesired background signal because of circulating liposomes, resulting in a suboptimal target-to-background ratio. The use of flow suppression techniques potentially can solve this problem. However, low flow velocity, reduction of the blood $\mathrm{T}_{1}$-relaxation time by the liposomes, and the complex and unstructured vasculature of the tumor blood vessels makes flow-suppression unreliable in tumors. Additionally, control liposomes generally display different blood-clearance rates, complicating a straightforward comparison between targeted and control particles. Ideally, the nonbound fraction should be cleared from the blood stream as soon as sufficient liposomes have associated with the target site. This would significantly enhance the specificity of the targeted liposomes for molecular imaging of angiogenesis. Furthermore, this approach would enable investigations into the accumulation kinetics of targeted liposomes and the optimization of MR imaging protocols for their detection.

A strategy to achieve rapid clearance of nonbound liposomes from the vasculature was developed by van Tilborg et al. [70, 71]. The technology is based on a so-called avidin chase experiment. Avidin is a 66-kDa glycoprotein, which can bind four biotin molecules with high affinity $\left(\mathrm{Kd}<10^{-15} \mathrm{M}\right)$ [72]. Avidin alone displays a very short intrinsic blood-circulation half-life, accumulates in the liver, spleen and kidneys, and is subsequently cleared from all organs within a couple of days [72, 73]. The basic idea is to equip the liposomal contrast agent with a targeting ligand, as well as with biotin. After initial intravenous injection of the agent, the liposomes are allowed to specifically bind and accumulate at the desired target site. In a second step, avidin is injected which then binds with high affinity to the biotin on the liposomes. As a consequence, the avidin triggers fast clearance of the circulating, nontarget-associated liposomes from the blood. This clearance strategy has been previously successfully applied to radiolabeled antibodies [74, 75] and liposomes [76] for nuclear imaging, as well as to dendrimer-based [77] and albuminbased [14] agents for MR imaging.

The avidin-chase technology was shown to improve the specificity of in vivo molecular magnetic resonance imaging of angiogenesis using targeted multimodal RGD-conjugated liposomes [71]. For this purpose, the RGD-conjugated liposomes were additionally equipped with biotin- $\mathrm{PEG}_{2000^{-}}$ DSPE (RGD-biotin-liposomes). In Fig. 5, the experiments are summarized. Tumor-bearing mice were intravenously injected with either RGD-biotin-liposomes or biotin-liposomes. After $100 \mathrm{~min}$ of circulation time, the mice received an intravenous infusion of avidin over a period of approximately 15 min to induce rapid blood clearance of the liposomes. Mice not infused with avidin served as controls. The RGD-biotin-liposome injection resulted in contrast enhancement mainly in the rim of the tumor, in agreement with the expected distribution of angiogenic blood vessels (Fig. 5a). The enhancement pattern changed only slightly between the 90- and 150-min time points after injection of the liposomes. In contrast, when avidin was infused at 100 min after injection, the percentage of enhanced pixels in the tumor decreased and enhanced pixels became more localized in the rim of the tumor (Fig. 5b), which suggests that part of the enhancement observed at the 90-min time point was caused by circulating liposomes. In Fig. $5 \mathrm{c}$ and d, the time lines of enhancement are summarized for the different experimental groups. Without application of the avidin chase (Fig. 5c), enhancement after injection of RGDbiotin-liposomes as well as biotin-liposomes decreases only slightly over the 150-min experimental time. After application of the avidin chase (Fig. 5d), the enhancement induced by the nonspecific biotin-liposomes was reduced to almost base levels, while the enhancement by RGD-biotinliposomes was still significant, with a much higher target-tobackground ratio. The multimodal liposomes were equipped with a fluorescent lipid as well and subsequent ex vivo fluorescence microscopy confirmed above interpretations.

This clearance strategy can be used to study relative contributions of target-associated, extravasated, as well as circulating nonbound liposomes to the MRI contrast enhancement in the tumor tissue. The avidin-chase technology can be applied to remove the blood pool component in the contrast-enhanced MRI experiment where flow suppression is not sufficient, which opens exciting possibilities for studying detection limits and angiogenesis targeting kinetics of the liposomal contrast agents.

\section{Synergistic targeting for combined imaging and treatment}

Despite the major advances with the introduction of multimodal targeted liposomes, the modest amounts of targetassociated contrast material still remains an obstacle for widespread application of molecular imaging of tumor angiogenesis. Recently, Kluza et al. [78] introduced a novel concept which may, at least partly, help to alleviate the sensitivity and specificity problems. The idea was to involve several different molecular markers specific for angiogenesis in the targeting process. The activated endothelium of angiogenic blood vessels seems to be an excellent candidate for this synergistic targeting concept as 
a

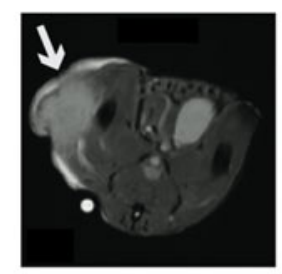

b

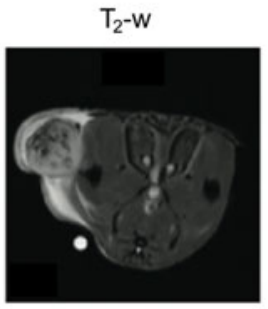

C

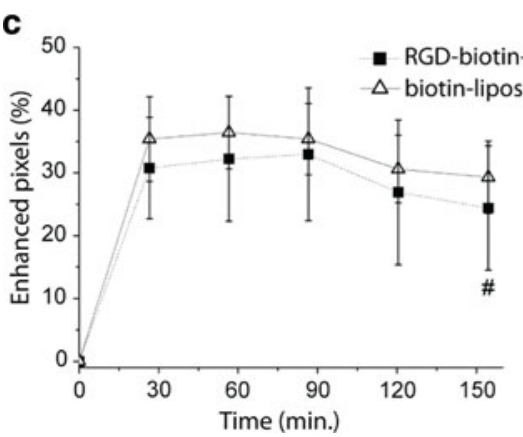

Fig. 5 Improved MR imaging of tumor angiogenesis by avidininduced clearance of nonbound liposomes. $\mathbf{a}, \mathbf{b} \mathrm{T}_{2}$-weighted $\left(\mathrm{T}_{2}-\mathrm{w}\right.$, left) and $\mathrm{T}_{1}$-weighted MR images (right) of a mouse with a B16F10 melanoma tumor in a subcutaneous position on the right flank (arrow). a Images of a mouse 90 and 150 min after intravenous injection of liposomes conjugated with both RGD peptide as well as biotin (RGD-biotin-liposomes). The significantly enhanced pixels are color coded according to the scale on the right. $\mathbf{b}$ Images of a mouse after intravenous injection of RGD-biotin-liposomes. Between the 90and 150-min time points, nonbound liposomes were removed by an

it over expresses a diverse set of molecules [79, 80]. Targeting of multiple receptors is relatively new in the field of molecular imaging and has been investigated before in a few studies only concerning drug delivery to cells [81, 82], for MR imaging of atherosclerosis [83], and for ultrasound imaging of tumor angiogenesis [84].

Moreover, for the first time Kluza et al. [78] showed that synergistic targeting with liposomes could be used to combine imaging and treatment of angiogenesis. This was achieved by using a unique combination of two targeting ligands, RGD and anginex (Anx), which bind specifically to two important angiogenic markers, $\alpha_{\mathrm{v}} \beta_{3}$ and galectin-1, respectively, and exert angiostatic activity as well $[7,85]$. Similar to aforementioned studies, the liposomes were of a bimodal nature containing a high payload of Gd-containing lipid for MR imaging and fluorescent lipids for fluorescent
$90 \mathrm{~min}$.

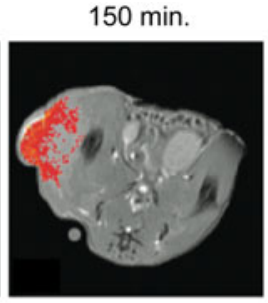

avidin infusion

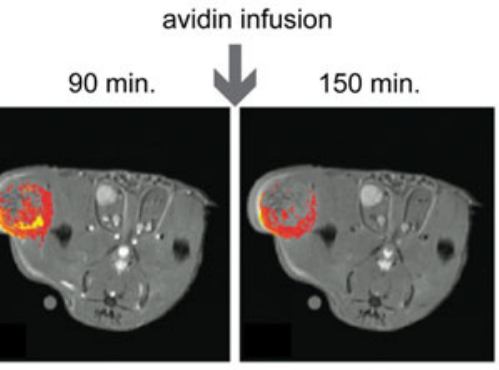

d

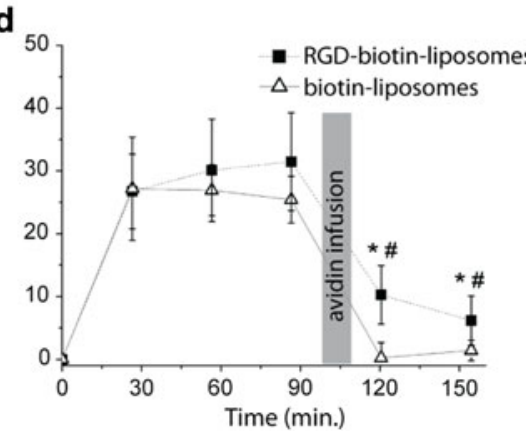

intravenous infusion of avidin, which binds with high affinity to the biotin on the liposomes and induces rapid blood clearance. c Time dependence of the percentage of enhanced pixels after injection with RGD-biotin-liposomes or liposomes with biotin only (biotin-liposomes). d Time dependence of the percentage of enhanced pixels after injection with RGD-biotin-liposomes and biotin-liposomes. After $100 \mathrm{~min}$ of circulation, the mice received and intravenous infusion of avidin over a period of approximately 15 min (gray bar) to induce clearance of nonbound liposomes. Figure adapted from Ref. [71] with permission

imaging, which enabled extensive evaluation and validation of the applied strategy.

The effects of simultaneous targeting of $\alpha_{\mathrm{v}} \beta_{3}$ and galectin-1 on the cellular uptake of the bimodal liposomes were investigated in vitro using human endothelial cells and the main results are summarized in Fig. 6. In the experiment, the targeting efficacy of the dual-targeted liposomes was compared to single-targeted liposomes to demonstrate the synergistic effect. Additionally, the difference between two dual-targeting strategies was studied. In the first approach, both RGD and Anx were conjugated to the same liposomes, whereas in the second, a mixture of single-conjugated RGD- and Anx-liposomes was used for targeting. Figure 6a schematically depicts the different experimental groups, which also included high and low concentrations of targeting peptides. Figure $6 \mathrm{~b}$ shows a 
quantitative comparison of the different targeting strategies after $3 \mathrm{~h}$ incubation at $37^{\circ} \mathrm{C}$. The quantitative concentration of Gd taken up by the cells provided an MRI-relevant indicator for the uptake of the paramagnetic liposomes. The highest uptake of Gd was observed for the Anx and RGD dual-conjugated liposomes with high peptide concentration (condition 5). The synergistic effect is clearly visible by comparing condition 5 with 7 . Incubations with a mixture of single-targeted RGD- and Anx-liposomes led to additive increase in Gd concentration when compared to the individual incubations (conditions 3 and 4). The dualtargeted liposomes (condition 5), however, resulted in approximately two-fold higher Gd uptake. Fluorescence microscopy provided additional evidence for the synergistic effect as shown in Fig. 6c. Highest levels of liposomal rhodamine fluorescence were observed in cells incubated with the dual-targeted liposomes (conditions 5 and 6) in agreement with the quantitative analyses.

Angiostatic potential of the RGD and Anx dual-conjugated liposomes was assessed by performing cell cycle analyses to investigate the antiproliferative effects induced by the synergistic effects of RGD and Anx. As readout to assess the therapeutic outcome, the percentages of cells in the $\mathrm{S}$ (DNA replication phase) and the G2/M (interphase/mitosis) phases were used. A decrease in $\mathrm{S}$ and $\mathrm{G} 2 / \mathrm{M}$ phase is generally considered a hallmark of impaired proliferation. A considerable reduction was found for treatments with the dual-targeted liposomes, a
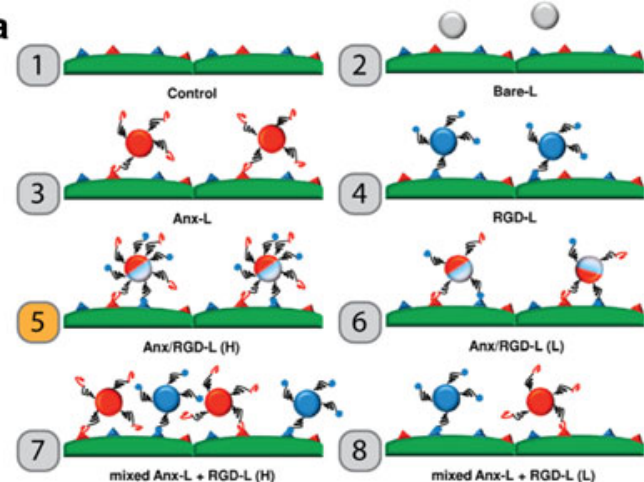

6
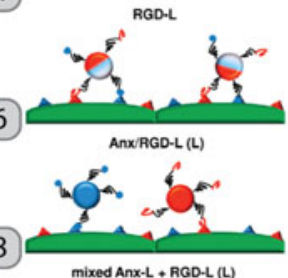

C

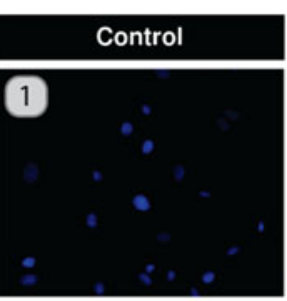

Anx/RGD-L (H)

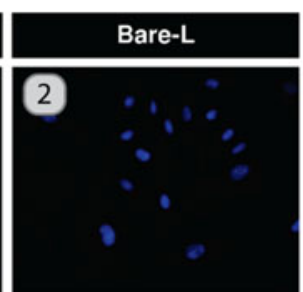

Anx/RGD-L (L)
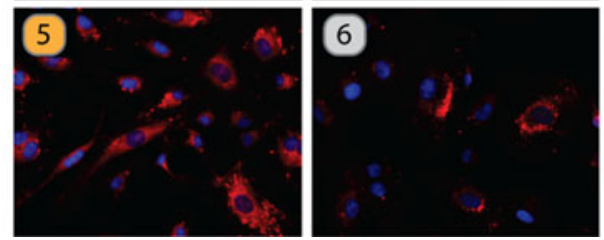

Fig. 6 Synergistic targeting of $\alpha_{\mathrm{v}} \beta_{3}$ integrin and galectin-1 with heteromultivalent paramagnetic liposomes for combined imaging and treatment of angiogenesis. a Schematic representation of the incubation schemes of in vitro human endothelial cells, for the following conditions: 1. Culture medium [Control]; 2. Nontargeted liposomes [Bare-L]; 3. Anx-conjugated liposomes [Anx-L]; 4. RGD-conjugated liposomes [RGD-L]; 5. Anx and RGD dual-conjugated liposomes containing high concentration of peptides [Anx/RGD L (H)]; 6. Anx and RGD dual-conjugated liposomes containing low concentration of peptides [Anx/RGD-L (L)]; 7. Mixture of Anx-L and RGD-L containing high concentration of peptides [mixed Anx-L + RGD-L $(\mathrm{H})]$; 8. Mixture of Anx-L and RGD-L containing low concentration of peptides [mixed Anx-L + RGD-L (L)]. High concentration of
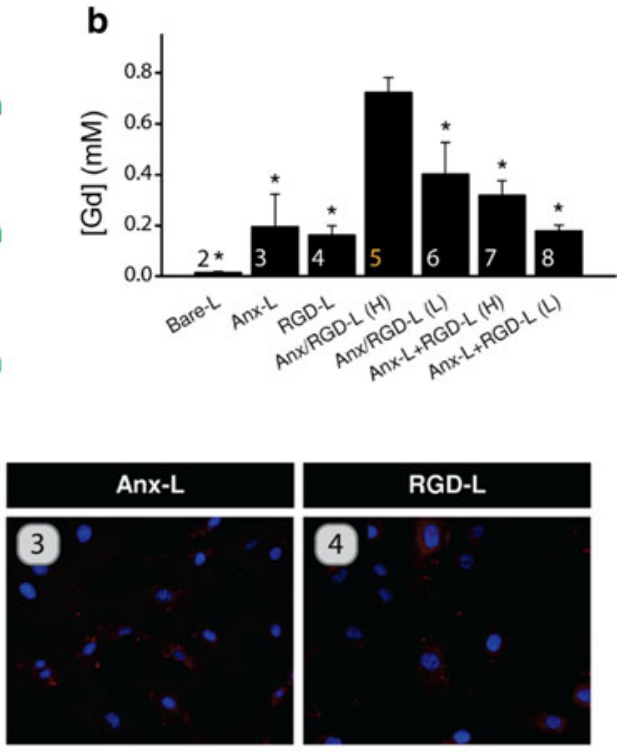

Anx-L+RGD-L (H)

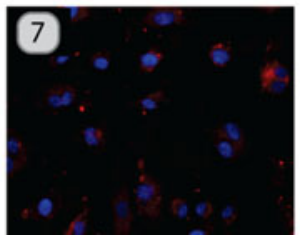

Anx-L+RGD-L (L)

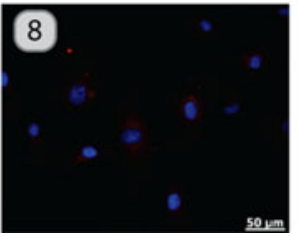

peptides $(\mathrm{H})$ were $10 \mu \mathrm{g} / \mu \mathrm{mol}$ lipid of Anx and $3 \mu \mathrm{g} / \mu \mathrm{mol}$ lipid of RGD, while low concentration (L) corresponded to $5 \mu \mathrm{g} / \mu \mathrm{mol}$ lipid of Anx and $1.5 \mu \mathrm{g} / \mu \mathrm{mol}$ lipid of RGD. b Gd uptake levels achieved with the different targeting strategies. Highest uptake of liposomal Gd was achieved for condition 5, demonstrating the synergistic effect of the two targeting ligands. c Fluorescence microscopy images of human endothelial cells incubated under the different conditions. Cell nuclei were stained with DAPI (blue fluorescence) and red fluorescence originated from the rhodamine-labeled liposomes. Highest cell associated red fluorescence was again observed for Anx and RGD dual-conjugated liposomes containing high concentration of peptides (condition 5). Adapted from Ref. [78] with permission 
which was stronger, however, not significantly, when compared to the single-conjugated liposomes. The synergistic effects of these dual-targeted liposomes create a great opportunity for their use in combined imaging and therapy of angiogenesis.

\section{Summary and perspective}

This paper reviewed our recent strategies on the design and application of multimodal targeted-liposomes for in vivo visualization, quantification, and treatment of tumor angiogenesis. As an angiogenesis-specific targeting ligand, we mainly used the cyclic RGD peptide, which binds with high specificity to the $\alpha_{v} \beta_{3}$ integrins expressed on angiogenic endothelium. Liposomes conjugated with a few hundred of these RGD peptides were shown to bind with great specificity to mouse tumor vascular endothelium. This enabled specific in vivo MRI visualization of angiogenic activity in tumors. Multimodality imaging properties were introduced by addition of fluorescent labels in the liposomes. This proved an essential tool for validation purposes, as it combined the best of both worlds - in vivo MR imaging of tumor angiogenesis with ex vivo fluorescence imaging of the liposomal accumulation and fate at the cellular level. Furthermore, the RGD-conjugated liposomes were applied for in vivo visualization of changes in tumor angiogenic activity after treatment with several angiostatic compounds.

The PEG-coated liposomes exhibit long in vivo blood circulation times. This can be exploited to increase the amount of contrast agent that accumulates at the angiogenic site. However, background MR signal from circulating liposomes in the blood pool reduces the target-to-background ratio. Incorporation of biotin-PEG-lipids in the bilayer was used for fast clearance of the liposomes from the blood pool using an avidin chase. This strategy improved the specificity of the MR readout and increased the target-to-background ratio dramatically. Finally, liposomes were equipped with two types of angiogenesis-specific ligands, i.e., RGD and anginex. First of all, this improved targeting efficacy by synergistic effects of the two targeting ligands. Second, the dual-targeted liposomes exhibited considerable angiostatic effects and therefore can be used for combined tumor angiogenesis imaging and therapy.

The reviewed work convincingly demonstrated that molecular MRI of angiogenesis and therapy monitoring in mouse models of cancer are feasible using the multimodal targeted liposomes. In recent years, several competitive platforms have been introduced in the field of nanoparticlebased molecular imaging. Some examples are iron-oxides [86], perfluorocarbon nanoparticles [63], polymeric nanocarriers [87], viruses [88], and high-density lipoproteinbased nanoparticles $[89,90]$. In our work, we have mainly focused on small peptides as targeting ligand for angiogenesis. Alternative ligands include antibodies and antibody fragments [91], small molecules [92], peptidomimetics [93], aptamers [94], and nanobodies [95]. This multi-disciplinary field of molecular imaging is evolving very fast and whether a given nanoparticle-ligand combination will be successful eventually remains to be evaluated for each particle separately.

Therefore, there is still a long way to go before this technology can be translated to human cancer. In particular, more knowledge is needed on the long-term fate of the bound and cell-internalized nanoparticles and on the organclearance kinetics and pathways in view of possible toxicity issues. Nevertheless, a lot of knowledge can be gained from preclinical studies in mouse models of cancer and for this the in vivo angiogenesis imaging technology proves a valuable tool already.

Acknowledgments This study was funded in part by the Integrated EU Project MEDITRANS (FP6-2004-NMP-NI-4/IP 026668-2), the European Community EC-FP6-project DiMI, LSHB-CT-2005512146 and by the BSIK program entitled Molecular Imaging of Ischemic Heart Disease (project number BSIK03033). This study was performed in the framework of the European Cooperation in the field of Scientific and Technical Research (COST) D38 Action MetalBased Systems for Molecular Imaging Applications.

Open Access This article is distributed under the terms of the Creative Commons Attribution Noncommercial License which permits any noncommercial use, distribution, and reproduction in any medium, provided the original author(s) and source are credited.

\section{References}

1. Kerbel RS (2008) Tumor angiogenesis. N Engl J Med 358:20392049

2. Ferrara N, Kerbel RS (2005) Angiogenesis as a therapeutic target. Nature 438:967-974

3. Kim KJ, Li B, Winer J et al (1993) Inhibition of vascular endothelial growth factor-induced angiogenesis suppresses tumour growth in vivo. Nature 362:841-844

4. Beliveau R, Gingras D, Kruger EA et al (2002) The antiangiogenic agent neovastat (AE-941) inhibits vascular endothelial growth factor-mediated biological effects. Clin Cancer Res $8: 1242-1250$

5. O'Reilly MS, Boehm T, Shing Y et al (1997) Endostatin: an endogenous inhibitor of angiogenesis and tumor growth. Cell 88:277-285

6. Benezra R, Rafii S (2004) Endostatin's endpoints-deciphering the endostatin antiangiogenic pathway. Cancer Cell 5:205-206

7. Griffioen AW, van der Schaft DW, Barendsz-Janson AF et al (2001) Anginex, a designed peptide that inhibits angiogenesis. Biochem J 354:233-242

8. Folkman J (2004) Endogenous angiogenesis inhibitors. APMIS 112:496-507

9. van der Schaft DW, Dings RP, de Lussanet QG et al (2002) The designer anti-angiogenic peptide anginex targets tumor endothelial cells and inhibits tumor growth in animal models. FASEB J 16:1991-1993 
10. De Schepper AM, Bloem JL (2007) Soft tissue tumors: grading, staging, and tissue-specific diagnosis. Top Magn Reson Imaging 18:431-444

11. He Q, Xu RZ, Shkarin P et al (2003) Magnetic resonance spectroscopic imaging of tumor metabolic markers for cancer diagnosis, metabolic phenotyping, and characterization of tumor microenvironment. Dis Markers 19:69-94

12. Zhou J, Lal B, Wilson DA et al (2003) Amide proton transfer (APT) contrast for imaging of brain tumors. Magn Reson Med 50:1120-1126

13. Kwee TC, Takahara T, Ochiai R et al (2008) Diffusion-weighted whole-body imaging with background body signal suppression (DWIBS): features and potential applications in oncology. Eur Radiol 18:1937-1952

14. Dafni H, Gilead A, Nevo N et al (2003) Modulation of the pharmacokinetics of macromolecular contrast material by avidin chase: MRI, optical, and inductively coupled plasma mass spectrometry tracking of triply labeled albumin. Magn Reson Med 50:904-914

15. Barrett T, Kobayashi H, Brechbiel M et al (2006) Macromolecular MRI contrast agents for imaging tumor angiogenesis. Eur $\mathbf{J}$ Radiol 60:353-366

16. Kiessling F, Morgenstern B, Zhang C (2007) Contrast agents and applications to assess tumor angiogenesis in vivo by magnetic resonance imaging. Curr Med Chem 14:77-91

17. Mulder WJ, Griffioen AW, Strijkers GJ et al (2007) Magnetic and fluorescent nanoparticles for multimodality imaging. Nanomedicine (Lond) 2:307-324

18. Glunde K, Pathak AP, Bhujwalla ZM (2007) Molecular-functional imaging of cancer: to image and imagine. Trends Mol Med 13:287-297

19. Sipkins DA, Cheresh DA, Kazemi MR et al (1998) Detection of tumor angiogenesis in vivo by $\alpha \mathrm{v} \beta 3$-targeted magnetic resonance imaging. Nat Med 4:623-626

20. Winter PM, Caruthers SD, Kassner A et al (2003) Molecular imaging of angiogenesis in nascent $\mathrm{Vx}-2$ rabbit tumors using a novel $\alpha \mathrm{v} \beta 3$-targeted nanoparticle and 1.5 tesla magnetic resonance imaging. Cancer Res 63:5838-5843

21. Morawski AM, Winter PM, Crowder KC et al (2004) Targeted nanoparticles for quantitative imaging of sparse molecular epitopes with MRI. Magn Reson Med 51:480-486

22. Mulder WJ, Strijkers GJ, Habets JW et al (2005) MR molecular imaging and fluorescence microscopy for identification of activated tumor endothelium using a bimodal lipidic nanoparticle. FASEB J 19:2008-2010

23. Lim EH, Danthi N, Bednarski M et al (2005) A review: Integrin $\alpha \mathrm{v} \beta 3$-targeted molecular imaging and therapy in angiogenesis. Nanomedicine 1:110-114

24. Mulder WJ, van der Schaft DW, Hautvast PA et al (2007) Early in vivo assessment of angiostatic therapy efficacy by molecular MRI. FASEB J 21:378-383

25. Zhang C, Jugold M, Woenne EC et al (2007) Specific targeting of tumor angiogenesis by RGD-conjugated ultrasmall superparamagnetic iron oxide particles using a clinical 1.5-T magnetic resonance scanner. Cancer Res 67:1555-1562

26. Schmieder AH, Caruthers SD, Zhang $\mathrm{H}$ et al (2008) Threedimensional MR mapping of angiogenesis with $\alpha 5 \beta 1(\alpha \mathrm{v} \beta 3)$-targeted theranostic nanoparticles in the MDA-MB-435 xenograft mouse model. FASEB J 22:4179-4189

27. Winter PM, Schmieder AH, Caruthers SD et al (2008) Minute dosages of $\alpha \mathrm{v} \beta 3$-targeted fumagillin nanoparticles impair $\mathrm{Vx}-2$ tumor angiogenesis and development in rabbits. FASEB $\mathbf{J}$ 22:2758-2767

28. Kiessling F, Huppert J, Zhang C et al (2009) RGD-labeled USPIO inhibits adhesion and endocytotic activity of $\alpha \mathrm{v} \beta 3$-integrin- expressing glioma cells and only accumulates in the vascular tumor compartment. Radiology 253:462-469

29. Soman NR, Baldwin SL, Hu G et al (2009) Molecularly targeted nanocarriers deliver the cytolytic peptide melittin specifically to tumor cells in mice, reducing tumor growth. J Clin Invest 119:2830-2842

30. Lijowski M, Caruthers S, Hu G et al (2009) High sensitivity: high-resolution SPECT-CT/MR molecular imaging of angiogenesis in the Vx2 model. Invest Radiol 44:15-22

31. Torchilin VP (2007) Targeted pharmaceutical nanocarriers for cancer therapy and imaging. AAPS J 9:E128-E147

32. Bangham AD, Standish MM, Watkins JC (1965) Diffusion of univalent ions across the lamellae of swollen phospholipids. J Mol Biol 13:238-252

33. Torchilin VP (2005) Recent advances with liposomes as pharmaceutical carriers. Nat Rev Drug Discov 4:145-160

34. Klibanov AL, Maruyama K, Torchilin VP et al (1990) Amphipathic polyethyleneglycols effectively prolong the circulation time of liposomes. FEBS Lett 268:235-237

35. Drummond DC, Meyer O, Hong K et al (1999) Optimizing liposomes for delivery of chemotherapeutic agents to solid tumors. Pharmacol Rev 51:691-743

36. Caride VJ, Sostman HD, Winchell RJ et al (1984) Relaxation enhancement using liposomes carrying paramagnetic species. Magn Reson Imaging 2:107-112

37. Navon G, Panigel R, Valensin G (1986) Liposomes containing paramagnetic macromolecules as MRI contrast agents. Magn Reson Med 3:876-880

38. Magin RL, Wright SM, Niesman MR et al (1986) Liposome delivery of NMR contrast agents for improved tissue imaging. Magn Reson Med 3:440-447

39. Koenig SH, Brown RD 3rd, Kurland R et al (1988) Relaxivity and binding of $\mathrm{Mn} 2+$ ions in solutions of phosphatidylserine vesicles. Magn Reson Med 7:133-142

40. Devoisselle JM, Vion-Dury J, Galons JP et al (1988) Entrapment of gadolinium-DTPA in liposomes. Characterization of vesicles by P-31 NMR spectroscopy. Invest Radiol 23:719-724

41. Unger E, Needleman P, Cullis P et al (1988) Gadolinium-DTPA liposomes as a potential MRI contrast agent. Work in progress. Invest Radiol 23:928-932

42. Tilcock C, Unger E, Cullis P et al (1989) Liposomal Gd-DTPA: preparation and characterization of relaxivity. Radiology 171: 77-80

43. Fossheim SL, Fahlvik AK, Klaveness J et al (1999) Paramagnetic liposomes as MRI contrast agents: influence of liposomal physicochemical properties on the in vitro relaxivity. Magn Reson Imaging 17:83-89

44. Fossheim SL, Il'yasov KA, Hennig J et al (2000) Thermosensitive paramagnetic liposomes for temperature control during MR imaging-guided hyperthermia: in vitro feasibility studies. Acad Radiol 7:1107-1115

45. Kabalka GW, Davis MA, Moss TH et al (1991) Gadoliniumlabeled liposomes containing various amphiphilic Gd-DTPA derivatives: targeted MRI contrast enhancement agents for the liver. Magn Reson Med 19:406-415

46. Kabalka GW, Davis MA, Holmberg E et al (1991) Gadoliniumlabeled liposomes containing amphiphilic Gd-DTPA derivatives of varying chain length: targeted MRI contrast enhancement agents for the liver. Magn Reson Imaging 9:373-377

47. Anelli PL, Lattuada L, Lorusso V et al (2001) Mixed micelles containing lipophilic gadolinium complexes as MRA contrast agents. Magn Res Mater Phys 12:114-120

48. Oliver M, Ahmad A, Kamaly N et al (2006) MAGfect: a novel liposome formulation for MRI labelling and visualization of cells. Org Biomol Chem 4:3489-3497 
49. Kamaly N, Kalber T, Ahmad A et al (2008) Bimodal paramagnetic and fluorescent liposomes for cellular and tumor magnetic resonance imaging. Bioconjug Chem 19:118-129

50. Mulder WJ, Strijkers GJ, Griffioen AW et al (2004) A liposomal system for contrast-enhanced magnetic resonance imaging of molecular targets. Bioconjug Chem 15:799-806

51. Strijkers GJ, Mulder WJ, van Heeswijk RB et al (2005) Relaxivity of liposomal paramagnetic MRI contrast agents. Magn Res Mater Phys 18:186-192

52. Hak S, Sanders HM, Agrawal P et al (2009) A high relaxivity Gd(III)DOTA-DSPE-based liposomal contrast agent for magnetic resonance imaging. Eur J Pharm Biopharm 72:397-404

53. Idee JM, Port M, Dencausse A et al (2009) Involvement of gadolinium chelates in the mechanism of nephrogenic systemic fibrosis: an update. Radiol Clin North Am 47:855-869 (vii)

54. Haubner R, Wester HJ, Weber WA et al (2001) Noninvasive imaging of $\alpha \mathrm{v} \beta 3$ integrin expression using 18F-labeled RGDcontaining glycopeptide and positron emission tomography. Cancer Res 61:1781-1785

55. Haubner R, Wester HJ, Burkhart F et al (2001) Glycosylated RGDcontaining peptides: tracer for tumor targeting and angiogenesis imaging with improved biokinetics. J Nucl Med 42:326-336

56. Janssen ML, Oyen WJ, Dijkgraaf I et al (2002) Tumor targeting with radiolabeled $\alpha \mathrm{v} \beta 3$ integrin binding peptides in a nude mouse model. Cancer Res 62:6146-6151

57. Chen X, Tohme M, Park R et al (2004) Micro-PET imaging of $\alpha \mathrm{v} \beta 3$-integrin expression with 18F-labeled dimeric RGD peptide. Mol Imaging 3:96-104

58. Chen X, Park R, Shahinian AH et al (2004) 18F-labeled RGD peptide: initial evaluation for imaging brain tumor angiogenesis. Nucl Med Biol 31:179-189

59. Haubner R (2006) $\alpha \mathrm{v} \beta 3$-integrin imaging: a new approach to characterise angiogenesis? Eur J Nucl Med Mol Imaging 33(Suppl 1):54-63

60. Chen X, Park R, Tohme M et al (2004) MicroPET and autoradiographic imaging of breast cancer $\alpha \mathrm{v}$-integrin expression using 18F- and 64Cu-labeled RGD peptide. Bioconjug Chem 15:41-49

61. Beer AJ, Schwaiger M (2008) Imaging of integrin $\alpha v \beta 3$ expression. Cancer Metastasis Rev 27:631-644

62. Caruthers SD, Cyrus T, Winter PM et al (2009) Anti-angiogenic perfluorocarbon nanoparticles for diagnosis and treatment of atherosclerosis. Wiley Interdiscip Rev Nanomed Nanobiotechnol 1:311-323

63. Kaneda MM, Caruthers S, Lanza GM et al (2009) Perfluorocarbon nanoemulsions for quantitative molecular imaging and targeted therapeutics. Ann Biomed Eng 37:1922-1933

64. Griffioen AW, Molema G (2000) Angiogenesis: potentials for pharmacologic intervention in the treatment of cancer, cardiovascular diseases, and chronic inflammation. Pharmacol Rev 52:237-268

65. Ruoslahti E, Pierschbacher MD (1986) Arg-Gly-Asp: a versatile cell recognition signal. Cell 44:517-518

66. Thumshirn G, Hersel U, Goodman SL et al (2003) Multimeric cyclic RGD peptides as potential tools for tumor targeting: solidphase peptide synthesis and chemoselective oxime ligation. Chemistry 9:2717-2725

67. Kok MB, Hak S, Mulder WJ et al (2009) Cellular compartmentalization of internalized paramagnetic liposomes strongly influences both T1 and T2 relaxivity. Magn Reson Med 61:1022-1032

68. Strijkers GJ, Hak S, Kok MB et al (2009) Three-compartment T1 relaxation model for intracellular paramagnetic contrast agents. Magn Reson Med 61:1049-1058

69. Koning GA, Schiffelers RM, Wauben MH et al (2006) Targeting of angiogenic endothelial cells at sites of inflammation by dexamethasone phosphate-containing RGD peptide liposomes inhibits experimental arthritis. Arthritis Rheum 54:1198-1208
70. van Tilborg GA, Strijkers GJ, Pouget EM et al (2008) Kinetics of avidin-induced clearance of biotinylated bimodal liposomes for improved MR molecular imaging. Magn Reson Med 60:1444-1456

71. van Tilborg GA, Mulder WJ, van der Schaft DW et al (2008) Improved magnetic resonance molecular imaging of tumor angiogenesis by avidin-induced clearance of nonbound bimodal liposomes. Neoplasia 10:1459-1469

72. Schechter B, Silberman R, Arnon R et al (1990) Tissue distribution of avidin and streptavidin injected to mice. Effect of avidin carbohydrate, streptavidin truncation and exogenous biotin. Eur J Biochem 189:327-331

73. Rosebrough SF, Hartley DF (1996) Biochemical modification of streptavidin and avidin: in vitro and in vivo analysis. $\mathrm{J}$ Nucl Med 37:1380-1384

74. Sinitsyn VV, Mamontova AG, Checkneva YY et al (1989) Rapid blood clearance of biotinylated IgG after infusion of avidin. J Nucl Med 30:66-69

75. Kobayashi H, Sakahara H, Hosono $M$ et al (1994) Improved clearance of radiolabeled biotinylated monoclonal antibody following the infusion of avidin as a "chase" without decreased accumulation in the target tumor. J Nucl Med 35:1677-1684

76. Laverman P, Zalipsky S, Oyen WJ et al (2000) Improved imaging of infections by avidin-induced clearance of $99 \mathrm{mTc}$-biotin-PEG liposomes. J Nucl Med 41:912-918

77. Kobayashi H, Kawamoto S, Star RA et al (2003) Activated clearance of a biotinylated macromolecular MRI contrast agent from the blood pool using an avidin chase. Bioconjug Chem 14:1044-1047

78. Kluza E, van der Schaft DW, Hautvast PA et al (2010) Synergistic targeting of $\alpha \mathrm{v} \beta 3$ integrin and galectin-1 with heteromultivalent paramagnetic liposomes for combined MR imaging and treatment of angiogenesis. Nano Lett 10:52-58

79. Adams RH, Alitalo K (2007) Molecular regulation of angiogenesis and lymphangiogenesis. Nat Rev Mol Cell Biol 8:464-478

80. Avraamides CJ, Garmy-Susini B, Varner JA (2008) Integrins in angiogenesis and lymphangiogenesis. Nat Rev Cancer 8:604-617

81. Laginha K, Mumbengegwi D, Allen T (2005) Liposomes targeted via two different antibodies: assay, B-cell binding and cytotoxicity. Biochim Biophys Acta 1711:25-32

82. Saul JM, Annapragada AV, Bellamkonda RV (2006) A dualligand approach for enhancing targeting selectivity of therapeutic nanocarriers. J Control Release 114:277-287

83. McAteer MA, Schneider JE, Ali ZA et al (2008) Magnetic resonance imaging of endothelial adhesion molecules in mouse atherosclerosis using dual-targeted microparticles of iron oxide. Arterioscler Thromb Vasc Biol 28:77-83

84. Willmann JK, Lutz AM, Paulmurugan R et al (2008) Dual-targeted contrast agent for US assessment of tumor angiogenesis in vivo. Radiology 248:936-944

85. Westlin WF (2001) Integrins as targets of angiogenesis inhibition. Cancer J 7(Suppl 3):S139-S143

86. Josephson L, Tung CH, Moore A et al (1999) High-efficiency intracellular magnetic labeling with novel superparamagnetic-Tat peptide conjugates. Bioconjug Chem 10:186-191

87. Levine DH, Ghoroghchian PP, Freudenberg J et al (2008) Polymersomes: a new multi-functional tool for cancer diagnosis and therapy. Methods 46:25-32

88. Manchester M, Singh P (2006) Virus-based nanoparticles (VNPs): platform technologies for diagnostic imaging. Adv Drug Deliv Rev 58:1505-1522

89. Cormode DP, Jarzyna PA, Mulder WJ et al (2010) Modified natural nanoparticles as contrast agents for medical imaging. Adv Drug Deliv Rev 62:329-338

90. Skajaa T, Cormode DP, Falk E et al (2010) High-density lipoprotein-based contrast agents for multimodal imaging of atherosclerosis. Arterioscler Thromb Vasc Biol 30:169-176 
91. Tada H, Higuchi H, Wanatabe TM et al (2007) In vivo real-time tracking of single quantum dots conjugated with monoclonal antiHER2 antibody in tumors of mice. Cancer Res 67:1138-1144

92. Pasqualini R, Koivunen E, Ruoslahti E (1997) Alpha v integrins as receptors for tumor targeting by circulating ligands. Nat Biotechnol 15:542-546

93. Hood JD, Bednarski M, Frausto R et al (2002) Tumor regression by targeted gene delivery to the neovasculature. Science 296:2404-2407
94. Farokhzad OC, Karp JM, Langer R (2006) Nanoparticle-aptamer bioconjugates for cancer targeting. Expert Opin Drug Deliv 3:311-324

95. Muyldermans S, Baral TN, Retamozzo VC et al (2009) Camelid immunoglobulins and nanobody technology. Vet Immunol Immunopathol 128:178-183 